\title{
De la nécessité d'une recherche de proportionnalité des solutions de gestion des déchets radioactifs à leur dangerosité
}

\author{
J.-M. Hoorelbeke* \\ Andra, 1-7 rue Jean-Monnet, 92298 Châtenay Malabry Cedex, France.
}

Reçu le 11 juin 2018 / Accepté le 6 juillet 2018

\begin{abstract}
Résumé - Une grande part des déchets radioactifs français disposent de filières d'élimination opérationnelles ou en cours de développement. Néanmoins, certains déchets interpellent aujourd'hui sur les modalités d'une gestion à long terme justement proportionnée à leur dangerosité, qu'il s'agisse des grands volumes de déchets de très faible activité qui seront produits par les démantèlements à venir ou de déchets aux caractéristiques intermédiaires, comme les déchets dits de faible activité à vie longue. La recherche de solutions de stockage s'inscrivant dans l'approche graduée recommandée par l'AIEA passe par une meilleure compréhension de la dangerosité des différents déchets et par une analyse des justes besoins en confinement et en isolement vis-à-vis de l'homme et de la biosphère. À ces besoins, pourront être associés des caractéristiques de barrières de long terme et des durées sur lesquelles les performances de ces barrières pourront être raisonnablement évaluées.
\end{abstract}

Mots clés : activité / approche graduée / classification / confinement / dangerosité / déchets radioactifs / filières de gestion / intrusion humaine / isolation / long terme / période radioactive / proportionnalité / stockage

\begin{abstract}
The search for proportionality of radioactive waste management solutions to their harmfulness. A large part of French radioactive waste benefit from disposal routes that are operational or under development. However, some types of waste are now questioning their long-term management modalities with a view to proportionality to their harmfulness: waste with a very low activity level to be generated by future decommissioning and dismantling, wastes with intermediate characteristics such as "low activity level and long-lived waste". The search for disposal solutions in line with the graded approach recommended by the IAEA requires a better understanding of the harmfulness of waste and an analysis of the reasonable need for containment and isolation from man and the biosphere. This need results in appropriate characteristics of long-term barriers and in durations on which the performance of these barriers can be assessed.
\end{abstract}

Keywords: activity level / classification / containment / disposal / graduate approach / human intrusion / harmfulness / isolation / long term / waste management route / radioactive half-life / radioactive waste / proportionality

\section{Introduction}

Tous les déchets radioactifs français disposent en principe d'un dispositif d'élimination industriel ou d'une voie tracée pour leur gestion à long terme (Fig. 1). Cela résulte, d'une part, de la création par la loi du 30 décembre 1991 de l'Agence nationale pour les déchets radioactifs (Andra) en tant qu'établissement public industriel et commercial et, d'autre part, de la mise en place de filières de gestion adaptées à chaque catégorie de déchets dans le cadre du Plan national de

\footnotetext{
* Auteur de correspondance :

jean-michel.hoorelbeke@andra.fr
}

gestion des matières et des déchets radioactifs (PNGMDR) (Legifrance, 1991; ASN, 2016). Ce plan mis à jour tous les trois ans a été publié pour la première fois en mai 2007 en application de la loi du 28 juin 2006 (Legifrance, 2006). Il se fonde notamment sur l'inventaire national des matières et déchets radioactifs de l'Andra (Andra, 2015).

En application du code de l'environnement, la définition de filières de gestion des déchets radioactifs doit en priorité prévenir et réduire la production et la nocivité des déchets radioactifs, et, pour les déchets radioactifs produits, privilégier dans l'ordre: le tri en vue de la réutilisation, le recyclage, la valorisation et au final le stockage des déchets ultimes (ASN, 2016). Le stockage consiste à placer les déchets radioactifs dans une installation spécialement aménagée pour les 


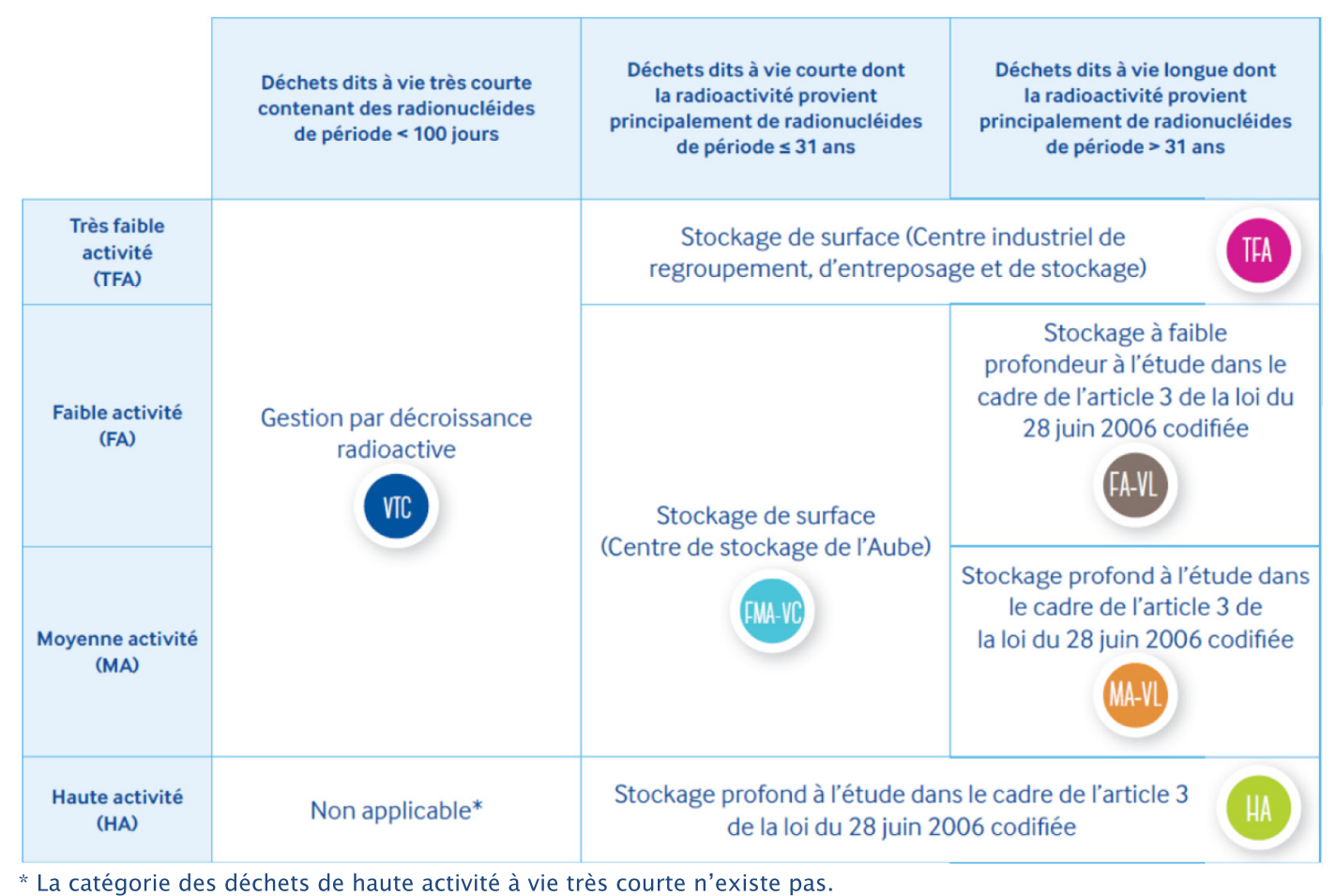

Figure 1. Classification des déchets radioactifs et filières de gestion associées.

conserver de façon potentiellement définitive, sans intention de les retirer ultérieurement.

Le système français de gestion des déchets radioactifs, dont on peut apprécier tous les bénéfices depuis des années, comporte néanmoins des incertitudes pour l'avenir. Les déchets radioactifs des différents types représentent des volumes significatifs alors même que les sites de stockage sont limités en nombre et en capacité. Il apparaît donc important de questionner la problématique du stockage des déchets radioactifs en France sous l'angle de la proportionnalité aux enjeux, c'est-à-dire la recherche d'une juste proportionnalité entre la dangerosité des déchets et les solutions de gestion. Une telle recherche est de nature à répondre aux recommandations de l'Agence internationale de l'énergie atomique (AIEA): «Conformément à l'approche graduée requise par les Normes fondamentales internationales et d'autres normes, la capacité du système de stockage définitif choisi de confiner les déchets et de les isoler des personnes et de l'environnement doit être proportionnée au risque que présentent les déchets » (AIEA, 2011).

Après avoir présenté de façon succincte les différentes filières de déchets radioactifs, cet article se propose de faire le point sur les difficultés rencontrées (déchets à la frontière entre catégories, déchets à risques radiologique et non radiologique...) et sur les possibilités de gestion proportionnée aux enjeux. Il s'inscrit dans la politique éditoriale de Radioprotection (Bourguignon et al., 2017).

\section{Classification des déchets radioactifs en France et filières de gestion}

La classification française des déchets radioactifs et les différentes filières qui lui sont associées sont présentées sur la
Figure 1. Les déchets dont la période radioactive est très courte, inférieure à 100 jours, sont gérés par décroissance sur leur site de production. En effet, leur activité diminue rapidement (d'un facteur 1024 au bout de 10 périodes, soit moins de 3 ans). Les considérer ensuite comme des déchets conventionnels constitue une bonne mesure de proportionnalité.

Les déchets dont la période est supérieure à 100 jours font ou feront l'objet d'un stockage, leur destination précise dépendant a priori de leur catégorie:

- les déchets de très faible activité (TFA) sont stockés en surface au Centre industriel de regroupement, d'entreposage et de stockage (Cires) (Fig. 2), installation classée pour la protection de l'environnement (ICPE) sise dans l'Aube. Leur activité massique moyenne est de l'ordre de $10 \mathrm{~Bq} / \mathrm{g}$;

- les déchets dits de faible et moyenne activité à vie courte (FMA-VC), contenant principalement des radionucléides de période inférieure à 31 ans, sont pris en charge, au fur et à mesure de leur production et de leur conditionnement, par le Centre de stockage de l'Aube (CSA, Fig. 3), installation nucléaire de base en surface, également établie dans l'Aube. Le CSA a accueilli entre 1991 et 2015, $304451 \mathrm{~m}^{3}$ de colis de déchets, pour une capacité totale d'un million de mètres cubes. Leur activité massique moyenne est de l'ordre de $10^{4} \mathrm{~Bq} / \mathrm{g}$ au bout d'une centaine d'années, puis moins de $10^{3} \mathrm{~Bq} / \mathrm{g}$ après un millier d'années;

- les déchets de moyenne activité à vie longue (MA-VL) et les déchets de haute activité (HA) constituent l'inventaire prévisionnel du projet Cigéo de stockage en formation géologique profonde (Fig. 4). L'activité massique moyenne des déchets MA-VL après 100 ans est supérieure à $10^{7} \mathrm{~Bq} / \mathrm{g}$, elle s'étage entre quelques $\mathrm{MBq} / \mathrm{g}$ et quelques centaines 


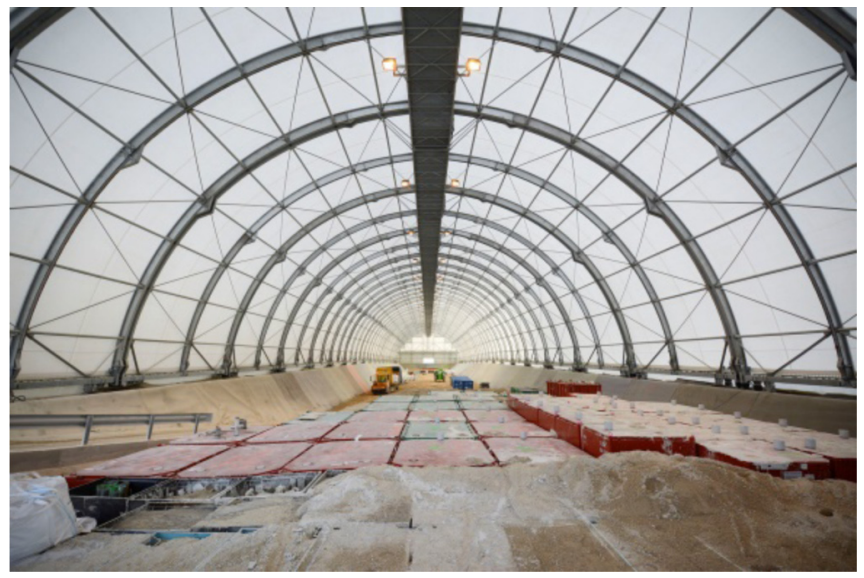

Figure 2. Stockage de déchets TFA au Cires (Aube).

de $\mathrm{MBq} / \mathrm{g}$ selon les familles de déchets. Elle est encore de l'ordre de $10^{6} \mathrm{~Bq} / \mathrm{g}$ après $10000 \mathrm{ans}$. Celle des déchets HA est supérieure à $10^{9} \mathrm{~Bq} / \mathrm{g}$ à 100 ans et de l'ordre de $10^{7} \mathrm{~Bq} / \mathrm{g}$ à 10000 ans. Les déchets déjà produits (qui représentent près de $70 \%$ des déchets MA-VL prévus d'être accueillis par Cigéo et plus de $30 \%$ des déchets HA) sont entreposés dans l'attente de leur stockage ; l'inventaire prévisionnel de Cigéo comporte de l'ordre de $73500 \mathrm{~m}^{3}$ de déchets MA-VL et $10000 \mathrm{~m}^{3}$ de déchets HA;

- enfin, la loi de programme du 28 juin 2006 stipule dans son article 4 la mise en place d'un programme de recherche et d'étude venant en sus des études et recherches sur «la gestion des déchets radioactifs à vie longue de haute ou de moyenne activité » et dont l'un des objectifs est « la mise au point de solutions de stockage pour les déchets graphites et les déchets radifères ». Ceux-ci sont rattachés dans la classification française aux déchets de faible activité à vie longue (FA-VL), c'est-à-dire dont la faible radioactivité provient principalement de nucléides de période supérieure à 31 ans. La classification associe cette catégorie de déchets à un stockage à faible profondeur.

\section{Des filières sous enjeux?}

La classification des déchets radioactifs présentée sur la Figure 1 est effectuée selon les deux axes d'entrée que sont la période radioactive et l'activité :

- la période radioactive de 31 ans est basée sur celle de deux produits de fission importants de la filière nucléaire, le césium 137 de période 30,1 ans et le strontium 90 de période 29,1 ans. Cette séparation bien opérationnelle correspond à la réalité de nombre de déchets radioactifs à gérer, même si les radionucléides à période courte sont en général accompagnés dans les déchets de radionucléides à période plus longue;

- la classification selon le niveau d'activité (TFA, FA, MA et HA) est plus subjective comme on a pu le voir plus haut. D'une part, il s'agit d'ordres de grandeur, d'autre part, les becquerels de différents radionucléides à période longue ne sont pas équivalents en termes de risque pour l'homme et l'environnement.

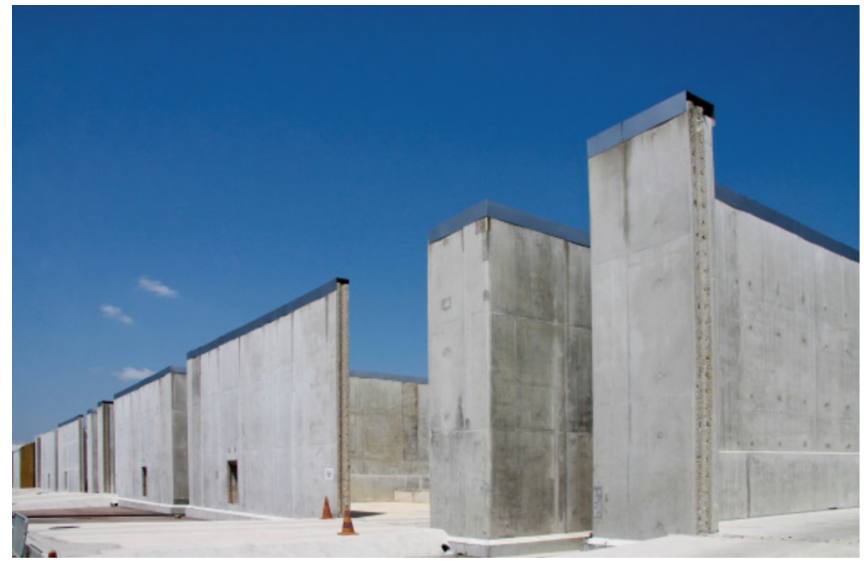

Figure 3. Ouvrages de stockage en surface du CSA (Aube).

Si cette classification présente le mérite de la simplicité, elle masque néanmoins des situations parfois plus complexes.

En effet, au-delà des déchets dont les conditions de gestion à long terme sont aujourd'hui bien définies (déchets à vie très courte, TFA et FMA-VC), d'autres interpellent autour de la question : « comment définir des solutions de gestion justement proportionnées à leur dangerosité ?».

Il s'agit d'abord d'un ensemble de substances dont le niveau d'activité se situe entre les déchets stockés au CSA et ceux a priori destinés à Cigéo. La plupart sont des déchets FAVL pour lesquels l'Andra mène des études conceptuelles de stockage à faible profondeur et explore au plan géologique un site potentiel d'accueil dans l'Aube.

Cette catégorie recouvre une grande diversité de types de déchets aux propriétés radiologiques et physico-chimiques variées :

- les déchets radifères et uranifères présentent une activité massique moyenne entre 100 et $10^{3} \mathrm{~Bq} / \mathrm{g}$. À la différence des déchets accueillis au CSA, ils décroissent plus lentement ou ne décroissent pas; les déchets contenant essentiellement de l'uranium voient leur activité croître légèrement dans le temps du fait de la génération de radium de filiation. Les déchets radifères sont majoritairement issus de matières premières naturellement riches en radionucléides naturels mais qui ne sont pas utilisées pour leurs propriétés radioactives (monazite, zircone).

- un autre ensemble de déchets FA-VL est constitué de déchets de graphite activés issus du fonctionnement et du futur démantèlement des réacteurs de la filière uranium naturel graphite gaz (UNGG), première génération de réacteurs électronucléaires français, et d'effluents radioactifs d'installations nucléaires de base enrobés dans du bitume. Ces déchets présentent un niveau d'activité massique moyen autour de $10^{5} \mathrm{~Bq} / \mathrm{g}$, et une décroissance vers $10^{4} \mathrm{~Bq} / \mathrm{g}$ à l'horizon de 10000 ans. Les volumes totaux de déchets FAVL considérés aujourd'hui sont de l'ordre de $250000 \mathrm{~m}^{3}$, ils se situent entre les volumes de déchets destinés respectivement à Cigéo et au CSA. Si une partie de ces déchets est déjà produite et entreposée, d'autres renvoient à des horizons nettement plus lointains pour leur stockage, en particulier les déchets de graphite générés par des démantèlements qui ne sont pas envisagés avant plusieurs décennies. 


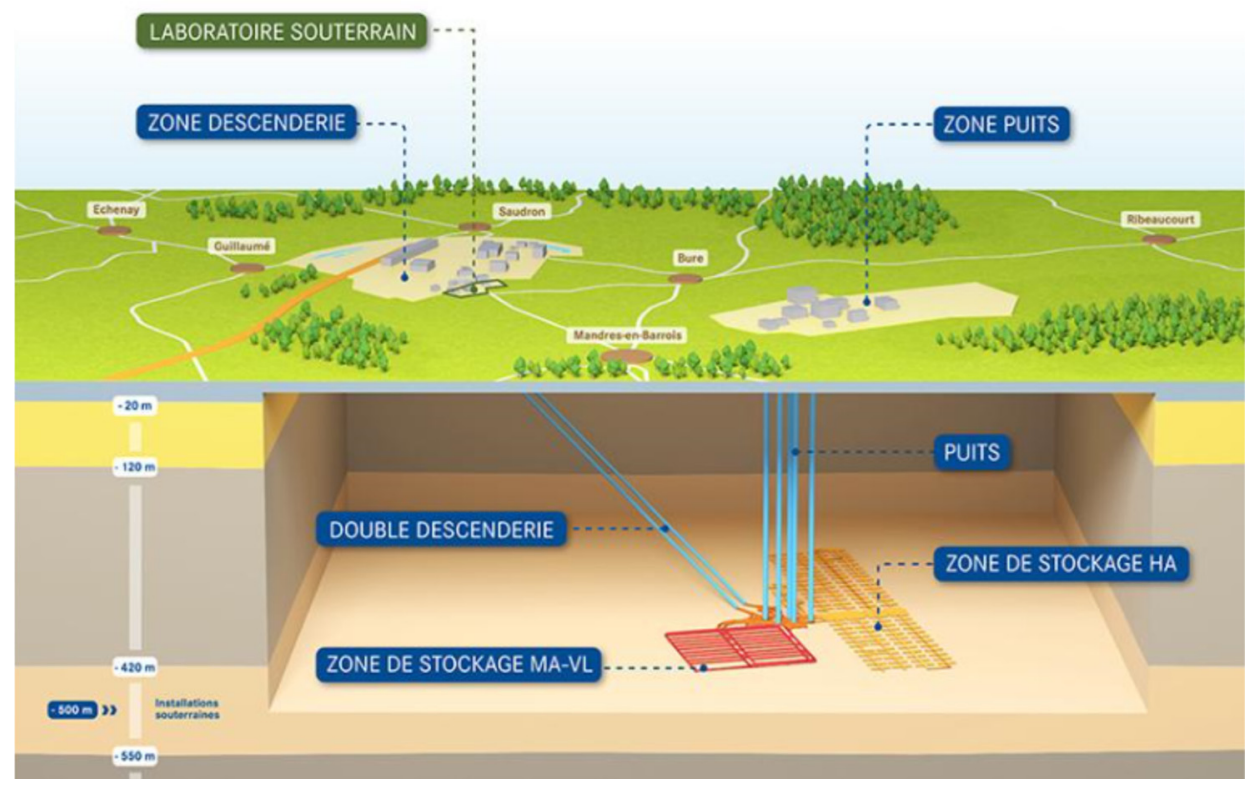

Figure 4. Illustration du projet Cigéo (Meuse/Haute-Marne).

Une problématique similaire pourra concerner d'autres substances que les déchets FA-VL, particulièrement les matières uranifères et thorifères que l'on considère aujourd'hui comme susceptibles d'être valorisées, mais qui, à terme, pourraient être requalifiées comme des déchets en fonction de l'évolution future de la politique énergétique: uranium appauvri issu de l'enrichissement de l'uranium naturel, uranium de retraitement... Le PNGMDR 2016-2018 confie à l'Andra une étude prospective du stockage éventuel de ces matières, en lien avec leurs propriétaires.

Par ailleurs, à la borne inférieure des déchets radioactifs du point de vue de leur dangerosité, les déchets TFA interpelleront également à terme sur les modalités de leur gestion. En effet, les futures opérations de démantèlement génèreront des volumes croissants de tels déchets, alors que les capacités de stockage actuellement disponibles au Cires sont limitées. Volumes futurs et niveaux d'activités associés dépendront notamment des politiques d'assainissement qui seront mises en œuvre. Les principes de conception des ouvrages de stockage du Cires sont comparables à ceux d'une installation de stockage de déchets conventionnels dangereux. Or une partie des déchets qui sont stockés au Cires sont à la fois non dangereux d'un point de vue non radiologique et d'un niveau d'activité très bas, potentiellement compatible avec les seuils de libération définis par l'AIEA et mis en œuvre dans certains pays: de 0,1 à $10000 \mathrm{~Bq} / \mathrm{g}$ pour les radionucléides artificiels, selon les radionucléides (exemples : $0,1 \mathrm{~Bq} / \mathrm{g}$ pour ${ }^{137} \mathrm{Cs} ; 1 \mathrm{~Bq} / \mathrm{g}$ pour ${ }^{90} \mathrm{Sr}$ ) et $1 \mathrm{~Bq} / \mathrm{g}$ pour les radioéléments à l'exception $\mathrm{de}^{40} \mathrm{~K}(10 \mathrm{~Bq} / \mathrm{g})(\mathrm{AIEA}, 2004)$. Si l'extension ou la création nouvelle de capacités de stockage du type Cires apparaît incontournable, la question se pose aussi de proposer des voies de gestion complémentaires pour la part des déchets TFA présentant la plus faible activité: valorisation ou recyclage, stockage à proximité des lieux de production selon des dispositions techniques et organisationnelles proportionnées à leur activité extrêmement faible...

\section{Entreposage et stockage}

Si les déchets en attente de stockage sont aujourd'hui entreposés de manière sûre, les installations d'entreposage n'offrent une protection de la santé et de l'environnement que sur une durée limitée, typiquement de plusieurs dizaines à une centaine d'années. Elles nécessitent le plus souvent des utilités actives comme une ventilation, une surveillance permanente et des activités régulières de maintenance.

Le principe d'un stockage consiste à conserver les déchets de manière sûre dans une installation $a d$ hoc sans limite de durée. L'installation de stockage est conçue de façon à pouvoir, à terme, maintenir une protection de la santé et l'environnement en l'absence de dispositifs actifs, voire même, selon la dangerosité des déchets, en cas de perte de contrôle institutionnel et de la mémoire collective de l'existence des déchets. Ses deux principales fonctions de sûreté sont ainsi progressivement assurées de manière de plus en plus passive: le confinement consiste à prévenir ou limiter la libération de substances toxiques et leur dispersion dans l'environnement; l'isolement consiste à séparer physiquement les déchets de l'environnement pour en rendre difficile l'accès aux personnes et les mettre à l'écart de phénomènes naturels d'altération atmosphérique et, sur le long terme, d'érosion. La mise en œuvre du stockage vise à réduire les charges indues transmises aux générations futures, sachant que ce sont notre génération et les précédentes qui ont bénéficié des activités à l'origine des déchets.

Dans une installation de stockage comme dans les installations d'entreposage et les autres installations nucléaires, les dispositifs de protection vis-à-vis des risques d'exposition sont optimisés en tenant compte des caractéristiques radiologiques des substances qui s'y trouvent. La conception des dispositifs de radioprotection d'une installation de stockage en exploitation ne se distingue pas dans le principe des autres types d'installations. La singularité d'une telle installation réside essentiellement dans le long terme: la 
question se pose non seulement des performances attendues des dispositifs qui devront fonctionner de manière passive, mais aussi de la durabilité à leur conférer en tenant compte de la décroissance radioactive des déchets, et de leur robustesse vis-à-vis de futurs évènements naturels ou anthropiques, probables ou hypothétiques.

Ainsi, le projet Cigéo interpose entre les déchets et les limites du système de stockage une épaisseur d'argilites très peu perméables d'une cinquantaine de mètres au moins, ce qui non seulement protège les déchets de l'eau et limite le relâchement des substances toxiques, mais aussi retarde et atténue considérablement la migration des substances dissoutes. La profondeur de plusieurs centaines de mètres protège durablement le système de stockage des évolutions naturelles en surface (la couche étudiée s'est formée il y a 160 millions d'années) et réduit fortement les risques d'intrusion involontaire après l'oubli hypothétique de l'existence de l'installation. Le confinement et l'isolement apportés par Cigéo sont étudiés sur une durée d'un million d'années. Leur haut niveau de performance et leur durabilité sont ainsi adaptés aux déchets HA et MA-VL.

Les ouvrages de stockage implantés en surface (CSA ou Cires) sont plus exposés à l'évolution naturelle. Ainsi, les échelles de temps qui peuvent être considérées sont moins longues. Les performances de confinement apportées par la couverture et le substratum argileux du Cires ou les ouvrages en béton du CSA sont également inférieures à celles de l'épaisse barrière argileuse de Cigéo. Leurs performances et leur durabilité sont adaptées à la moindre dangerosité des déchets pris en charge, tout en se révélant encore surdimensionnées pour les moins actifs des déchets TFA.

Dans ce contexte, comment gérer de manière juste des substances intermédiaires à la fois du point de vue de leurs contenus radiologiques et de leurs volumes? L'équité intergénérationnelle nous impose de leur trouver une solution de stockage suffisamment performante et durable au regard de leurs caractéristiques radiologiques, mais sans pénaliser inutilement la génération actuelle par une complexité technique ou une immobilisation de ressources injustifiées.

Comme indiqué plus haut, les prescriptions de sûreté émises par l'AIEA sur le stockage définitif des déchets radioactifs disposent que la capacité d'un système de stockage à confiner les déchets et à les isoler des personnes et de l'environnement doit être proportionnée au risque que présentent les déchets (en application de l'approche graduée encouragée par l'AIEA). L'Andra cherche ainsi à inscrire ses études de solutions de stockage dans une recherche de proportionnalité à la dangerosité des différents déchets. C'est cette dangerosité qui devrait alors déterminer les degrés de confinement et d'isolement des déchets à assurer, sans préjudice de l'obligation de rechercher les meilleures techniques disponibles en tenant compte des contraintes techniques et économiques.

\section{Apprécier la dangerosité des déchets radioactifs}

Parce que certains déchets radioactifs présentent aussi des risques non radiologiques liés à leur nature chimique, leur dangerosité doit rendre compte également de ces risques. Une approche de la dangerosité à la fois radiologique et non radiologique des déchets devrait aussi permettre une mise en regard des déchets radioactifs avec les déchets conventionnels et la prise en compte des pratiques environnementales hors du domaine nucléaire.

Un rapprochement peut être fait avec la demande exprimée par le PNGMDR 2016-2018 à l'Institut de radioprotection et de sûreté nucléaire (IRSN) de piloter une étude sur la notion de nocivité des matières et déchets radioactifs, notion peut-être plus large car devant intégrer des considérations sur l'évolution de la nocivité à court, moyen et long terme, sur l'écotoxicité et sur l'impact sur la santé humaine des modalités de gestion envisagées dans le PNGMDR. Cette demande faisait suite à l'avis de l'Autorité environnementale sur l'évaluation environnementale du PNGMDR 2016-2018.

Le risque radiologique dépend du niveau d'activité et des périodes des radionucléides en présence, des types de rayonnement et de leur énergie, ainsi que de propriétés chimiques qui impactent notamment la mobilité des radionucléides. Les valeurs d'activités massiques moyennes en $\mathrm{Bq} / \mathrm{g}$ données plus haut pour chaque catégorie de déchets doivent ainsi être fortement pondérées par les caractéristiques des différents radionucléides : deux déchets d'activités massiques similaires ne présentent pas la même dangerosité radiologique lorsque les principaux nucléides en présence diffèrent par leur caractère plus ou moins radiotoxique.

Il n'apparaît pas simple de définir une notion de risque ou de danger radiologique qui soit associée de manière intrinsèque à un déchet en préalable à la définition d'un mode de gestion. Cette difficulté peut être illustrée par la détermination par l'AIEA de valeurs seuils d'activité massique en vue de l'exclusion, l'exemption ou la libération de substances contenant des radionucléides artificiels. L'AIEA s'est dotée pour cela d'un jeu de situations typiques d'exposition qui présentent nécessairement un caractère conventionnel (mise en décharge, réutilisation dans une fonderie, mise au contact régulière, réutilisation pour la construction etc.) (AIEA, 2005). Puis, l'AIEA a effectué un calcul inverse sur la base d'une dose individuelle ajoutée de $10 \mu \mathrm{Sv} / \mathrm{an}$ qu'elle a considérée acceptable. Cette démarche introduit une forte dépendance des résultats au choix des valeurs de paramètres utilisées dans le calcul.

Face à la difficulté de caractériser le potentiel de danger intrinsèque d'un déchet radioactif, l'approche classique de la gestion à long terme des déchets, conforme aux recommandations de l'AIEA, repose sur l'évaluation du risque résiduel généré par les déchets placés dans une installation de stockage et sa comparaison aux objectifs de protection assignés à cette installation. L'adoption d'une démarche itérative faisant intervenir plusieurs concepts ou installations de stockage devrait alors permettre de trouver un optimum. Cette approche a néanmoins plusieurs limites vis-à-vis de l'objectif poursuivi. Partant de concepts de stockage préétablis, elle risque de freiner une recherche de solutions innovantes qui démarrerait des caractéristiques propres du déchet. Par ailleurs, les expositions résiduelles calculées correspondent le plus souvent à de faibles ou très faibles doses, dans un contexte incitant à l'adoption d'hypothèses conservatives et à la recherche d'expositions aussi faibles que possible. Si une telle approche peut en principe permettre de démontrer qu'une solution de stockage donnée offre des performances suffisantes au regard 
des caractéristiques du déchet, elle n'est pas nécessairement adaptée à en justifier la proportionnalité.

Pour ce qui concerne les risques non radiologiques, la réglementation applicable aux déchets conventionnels définit leur dangerosité, directe ou via des produits issus de leur altération comme une lixiviation, par les propriétés suivantes (UE, 2008) : explosif, comburant, inflammable, irritant, nocif ou toxique, c'est-à-dire pouvant entraîner des risques (respectivement limités ou graves, aigus, chroniques, voire mortels) pour la santé par inhalation, ingestion ou pénétration cutanée, cancérogène, corrosif en contact avec des tissus vivants, infectieux, toxique pour la reproduction, mutagène, (hyper) sensibilisant, écotoxique. Si les risques non radiologiques sont en général associés à des substances non radioactives contenues dans les déchets (comme des métaux lourds ou de l'amiante), certains radionucléides, particulièrement l'uranium, présentent une toxicité à la fois radiologique et chimique.

\section{Définir les performances et la durabilité attendues d'une installation de stockage proportionnée à la dangerosité des déchets}

Le confinement passif des déchets dans une installation de stockage repose sur les performances et la durabilité des colis de déchets, elles-mêmes dépendantes des conditions physicochimiques dans le stockage, la capacité à limiter la mobilité des substances toxiques dans le système de stockage (par exemple via le potentiel d'oxydo-réduction), la maîtrise des conditions hydrauliques (flux d'eau, phénomène de transport de masse prédominant), la capacité à retarder la migration des substances toxiques et à atténuer le flux sortant du système de stockage. Le degré de confinement renvoie notamment aux caractéristiques hydrodynamiques et à l'épaisseur des barrières (particulièrement celles de la formation géologique hôte ou de la couverture dans un stockage géologique ou à faible profondeur). La qualité du confinement est en premier lieu appréciée par les efforts mis en œuvre par le concepteur pour optimiser le système de stockage en recourant aux meilleures techniques disponibles. D'un point de vue plus quantitatif, les performances de confinement d'une installation de stockage peuvent être évaluées par des indicateurs tels que des débits molaires aux interfaces entre les différents compartiments du système de stockage, en accompagnant les calculs d'une analyse des incertitudes associées aux modèles mis en œuvre. Au final, l'acceptabilité du degré de confinement offert vis-àvis du risque radiologique est vérifiée par des calculs d'exposition individuelle sur le public et le respect de la contrainte de dose. Il peut s'y ajouter d'un point de vue environnemental une estimation du marquage des nappes; dès lors que ce marquage n'est pas relié à un impact sanitaire potentiel, la prescription d'un niveau acceptable constitue une interrogation en soi.

Une question essentielle à laquelle le concepteur d'une solution de stockage est confronté est la durée pendant laquelle le confinement doit être assuré et l'échelle de temps sur laquelle l'évaluation d'impact est menée. La décroissance radioactive aide dans certains cas à déterminer des durées pertinentes. Ainsi, lorsque le carbone 14 (période de 5300 ans), l'américium 241 (432,2 ans) ou le radium 226 (1600ans) constituent les radionucléides prédominants dans certains déchets de type FA-VL, une échelle de temps de l'ordre d'une à quelques dizaines de milliers d'années peut être considérée, justifiant, pour ces déchets, l'étude d'un stockage à faible profondeur. Cette échelle est à mettre en regard de celle de plusieurs centaines de milliers d'années envisagée pour les déchets HA. La question est plus délicate face à des déchets ou matières dont l'activité est faible et ne paraît pas justifier a priori un stockage en formation géologique profonde, mais dont la décroissance radioactive est faible elle-aussi ou inexistante. Un parallèle peut être établi entre cette problématique et la gestion des déchets conventionnels dangereux.

Si les incertitudes sur les modèles phénoménologiques utilisés pour évaluer les performances de confinement augmentent avec les échelles de temps, il existe un autre ensemble de paramètres qui pourrait y être sensible: il s'agit des facteurs permettant de passer d'une concentration de radionucléides (ou de toxiques chimiques) dans l'environnement à une exposition individuelle de personnes et de la contrainte de dose elle-même. À l'entrée dans l'anthropocène mais aussi au regard de l'évolution humaine passée, le réalisme d'hypothèses concernant les voies d'exposition extrapolées de la situation actuelle ou de situations passées diminue avec le temps. Face à cette difficulté, l'Autorité de sûreté nucléaire (ASN) prescrit qu'on «supposera la constance des caractéristiques de l'homme (sensibilité aux rayonnements, habitudes alimentaires, conditions de vie, connaissances générales actuelles, notamment dans les domaines technique et médical) » (ASN, 2008).

Les conditions géodynamiques du site et la profondeur de stockage interviennent fortement sur la durée de protection du système de stockage et sa capacité d'isolement.

L'altération et l'érosion, reliées aux évolutions climatiques (glaciation), affectent le stockage sur des échelles de temps d'autant moins longues qu'il est proche de la surface: augmentation progressive de l'accessibilité des déchets à l'homme, modification des conditions hydrauliques, diminution d'épaisseur de couverture jusqu'à éventuellement sa disparition complète. Seule une grande profondeur peut protéger les déchets de l'érosion sur des durées très étendues et permet d'exclure l'occurrence d'une situation de mise à nu des déchets à l'échelle du million d'années.

Pour évaluer la capacité d'isolement d'une solution de stockage, les prescriptions de sûreté de l'AIEA et les guides établis par l'ASN prévoient la prise en compte de situations d'intrusion humaine involontaire, après perte du contrôle constitutionnel supposé intervenir au bout de quelques siècles (300 à 500 ans). Ces situations sont examinées sous l'angle de la dégradation potentielle du confinement, pouvant aller jusqu'à une mise à nu de déchets bien avant les processus naturels, et celui de l'exposition individuelle des personnes directement impliquées dans l'intrusion.

En condition de stockage, la concentration des déchets en un même lieu tend nécessairement à augmenter localement le potentiel de danger, ce qui se traduit de manière négative dans une situation d'intrusion ou de mise à nu des déchets, que cette mise à nu soit d'origine naturelle ou anthropique. Ce détriment est néanmoins à mettre en regard des bénéfices qu'apporte le regroupement de déchets dans une installation de stockage: qualité du confinement, renforcement du 
contrôle et de la surveillance. Dans une recherche de proportionnalité, cela pose la question du rôle de situations d'intrusion ou de mise à nu vis-à-vis de la fixation de seuils d'activité massique pour l'acceptation des déchets, en fonction des échelles de temps. Il est à noter que les pratiques environnementales en dehors du domaine nucléaire ne considèrent pas ces types de situations.

\section{Conclusion}

En conclusion, la recherche d'une juste proportionnalité entre la dangerosité des déchets radioactifs et les solutions de gestion à long terme appelle des réflexions sur la façon de décrire cette dangerosité et sur celle d'appréhender la protection des générations futures et l'acceptabilité du risque résiduel sur de grandes échelles de temps. Si les connaissances du radioprotectionniste, du spécialiste des sciences de la terre et de l'ingénieur nucléaire permettent d'éclairer ces questionnements, leur instruction peut difficilement être circonscrite au sein de cette seule communauté, et semble mériter une plus large ouverture.

\section{Références}

AIEA. 2004. Application of the concepts of exclusion, exemption and clearance, safety guide $\mathrm{n}^{\circ}$ RS-G-17, AIEA, Vienne.

AIEA. 2005. Derivation of activity concentration values for exclusion, exemption and clearance, safety reports series $n^{\circ} 44$, AIEA, Vienne.

AIEA. 2011. Stockage définitif des déchets radioactifs, Prescriptions de sûreté particulières $n^{\circ}$ SSR-5, AIEA, Vienne.

Andra. 2015. Inventaire national des matières et déchets radioactifs. ASN. 2008. Orientations générales de sûreté en vue d'une recherche de site pour le stockage des déchets de faible activité massique à vie longue, Autorité de sûreté nucléaire.

ASN. 2016. Plan national de gestion des matières et des déchets radioactifs 2016-2018, Ministère de l'Environnement, de l'Énergie et de la Mer/Direction générale de l'énergie et du climat, Autorité de sûreté nucléaire.

Bourguignon M, Bérard P, Bertho JM, Farah J, Mercat C. 2017. What's next in Radioprotection? Radioprotection 52: 21-28.

Legifrance. 1991. Loi n 91-1381 du 30 décembre 1991 relative aux recherches sur la gestion des déchets radioactifs.

Legifrance. 2006. Loi $\mathrm{n}^{\circ} 2006-739$ du 28 juin 2006 de programme relative à la gestion durable des matières et déchets radioactifs.

UE. 2008. Directive européenne $n^{\circ} 2008 / 98 / C E$ du 19 novembre 2008 relative aux déchets et abrogeant certaines directives.

Citation de l'article : Hoorelbeke J-M. 2018. De la nécessité d'une recherche de proportionnalité des solutions de gestion des déchets radioactifs à leur dangerosité. Radioprotection 53(3): 167-173 